\title{
Design of 0.13um CMOS Low Voltage Rail to Rail Fully Differential Operational Amplifier - Review
}

\author{
Nikesh Hadke \\ Dept.of Elect \& Comm.Engg. \\ B.D.College of Engineering, \\ Sevagram, Wardha (M.S), \\ India
}

\author{
Prashant Indurkar \\ Dept.of Elect \& Comm.Engg. \\ B.D.College of Engineering, \\ Sevagram, Wardha (M.S), \\ India
}

\author{
Ravindra Kadam \\ Dept.of Elect \& Comm.Engg. \\ B.D.College of Engineering, \\ Sevagram, Wardha (M.S), \\ India
}

\begin{abstract}
An operational amplifier is one of the most commonly used components in analog and digital circuit designs. Low voltage and low power operational amplifier design has become an increasingly interesting subject as many applications switch to portable battery powered operations. The need for design techniques to allow amplifiers to maintain an acceptable level of performance when the supply voltages are decreased is immense. One of the most important features in low voltage amplifier designs is ensuring that the amplifier maintains constant behavior in the presence of rail-to-rail input common mode variations while providing a rail-to-rail output to maximize signal-to-noise ratio. As the supply voltage to a standard CMOS op-amp is reduced, the input common mode range and the output swing get reduced drastically. Special circuits have to be used to raise them up to rail-to-rail supply voltage. In this work we propose a design of a low-voltage CMOS rail-to-rail folded cascode operational amplifier to be realized in a standard $130 \mathrm{~nm}$ CMOS technology with $1.2 \mathrm{~V}$ supply voltage and consumes power less than $400 \mathrm{uW}$. A two stage miller compensated folded cascode op-amp has a UGB $>10 \mathrm{MHz}$ and achieves a gain $>80 \mathrm{~dB}$ over almost full range of the common mode input voltage.
\end{abstract}

\section{Keywords}

Rail to Rail, operational amplifier, folded cascode, CMOS, low voltage.

\section{INTRODUCTION}

An op-amp is a key building block in analog circuit design. It is a DC coupled high gain electronic voltage amplifier. An op- amp produces an output voltage that is typically hundreds of thousand times larger than the voltage difference between its input terminals. As the supply voltage and current of an analog circuit decrease there are certain performance measures of the circuit which will suffer a loss in performance. For example, for lower supply voltages the signal to noise ratio of the circuit will decrease, as the maximum input and output signal for the circuit will be smaller. Also the achievable bandwidth will be reduced as the supply voltage and total current are reduced. Less headroom tends to be available when the supply voltages are reduced. A reduction in headroom removes the possibility of using cascoded or stacked devices to increase the output impedance. In terms of the minimum usable supply voltage, it is required that the following expression is satisfied, VDD > Vsignal_swing $+\mathrm{K}^{*}$ VDsat, where VDsat is the minimum transistor saturation voltage, Vsignal_swing is the signal swing of the circuit, and $\mathrm{K}$ represents the number of transistors stacked in series. Thus, as the supply voltage decreases, the largest possible value of $\mathrm{K}$ will also decrease, which implies cascoding, may become difficult or impractical. Research indicates that major performance measures such as DC gain, GBW, and PM of an amplifier are greatly influenced by the design of the input stage. Because of this, there has been much focus on the optimal design of the input stage to keep these performance measures constant across the common-mode range. Most of the focus has shifted towards the argument of keeping gm constant, but the overall intent is to obtain a constant operation for all amplifier specifications. Many designs use the same architecture, but make changes to the input stage to obtain a rail-to-rail input stage and constant operation.

A commonly used architecture for low voltage amplifier designs is a folded cascode Amplifier. This architecture is used because it maximizes the ability to manipulate the input stage to achieve the rail-to-rail operation that is desired in low voltage design. A commonly used technique for the input stage is the implementation of a complementary input pair. This means that the input consists of an n-ch input pair as well as a p-ch input pair. Implementing this technique insures that at least one input pair is operating when the common-mode shifts near the supply rails. However, the use of complementary input pairs does not insure that there will be a constant operation across the common-mode range. Additional measures must be taken to insure constant operation. To achieve a rail-to-rail output for a low voltage amplifier, it is typical to implement a class-A output stage. A class-A output stage allows for the transient output signal to swing relatively close to the supply rails.

\section{OP-AMP DESIGN OBJECTIVES}

One of the most important features in low voltage amplifier designs is ensuring that the amplifier maintains constant behavior in the presence of rail-to-rail input common mode variations while providing a rail-to-rail output to maximize signal-to-noise ratio. The amplifier architecture aims at achieving constant amplifier operation over a rail-to-rail common-mode input voltage range. The concept of constant operation refers to the ability to maintain constant specifications such as gain, gain bandwidth product, phase margin, slew rate, and power consumption against large variations in input common-mode voltage. The proposed fully differential two stage miller compensated folded cascode opamp topology could be used in a very low voltage application with a typical supply voltage of $1.2 \mathrm{~V}$ making it ideal for the various applications where the power dissipation is an important specification for life time battery.

\section{TECHNIQUES FOR LOW VOLTAGE RAIL TO RAIL OP-AMP DESIGN}

There have been a number of works that have explored how to achieve a rail-to-rail behavior through proper input and output stage designs. Rail-to-rail input techniques mainly revolves around the idea of maintaining a constant input gm across the full common-mode range. Most popularly complementary input differential pair is used to design rail to rail input differential stage. Many schemes have been proposed to 
equalize gm-T, such as constant square root summation, for strong inversion region, constant current summation, for weak inversion region, transconductance compensation, feed forward, bulk-driven, and transconductance feedback techniques. To achieve rail-to-rail outputs, typically a class-A or class-AB output-stage is implemented. However, many different implementations exist for obtaining rail-to-rail input/outputs, each of which has their own advantages and disadvantage.

\subsection{Rail to Rail op-amp with Common-Mode Feed-Forward Circuit (CMFF)}

In this op-amp [1], rail-to-rail input operation is enabled by using complementary input pairs with gm control. To maximize the output swing a rail-to-rail output stage is employed. For low-voltage low-power operation, the operating transistors in the input and output stage are biased in the sub threshold region. A common-mode feed-forward circuit (CMFF) increases CMRR, reducing drastically the variations in the output common mode voltage and keeping the DC gain almost constant. This CMFF circuit biases conveniently the rail-to-rail outputs so that the output common mode voltage $\mathrm{V} 0 \mid \mathrm{CM}$ is kept almost constant in spite of variations in the input common mode voltage $\mathrm{Vcm}$. The rail to rail output stage is implemented with classic common source configuration. Implemented with $0.18 \mu \mathrm{m}$ the op-amp achieves a DC open loop gain is $51 \mathrm{~dB}$, and the slew-rate is $0.04 \mathrm{~V} / \mu$ s with a $10 \mathrm{pF}$ capacitive load connected to each of the amplifier outputs. For the same load the unity gain frequency is $131 \mathrm{kHz}$ with a $64^{\circ}$ phase margin.

Fig.1 (a)

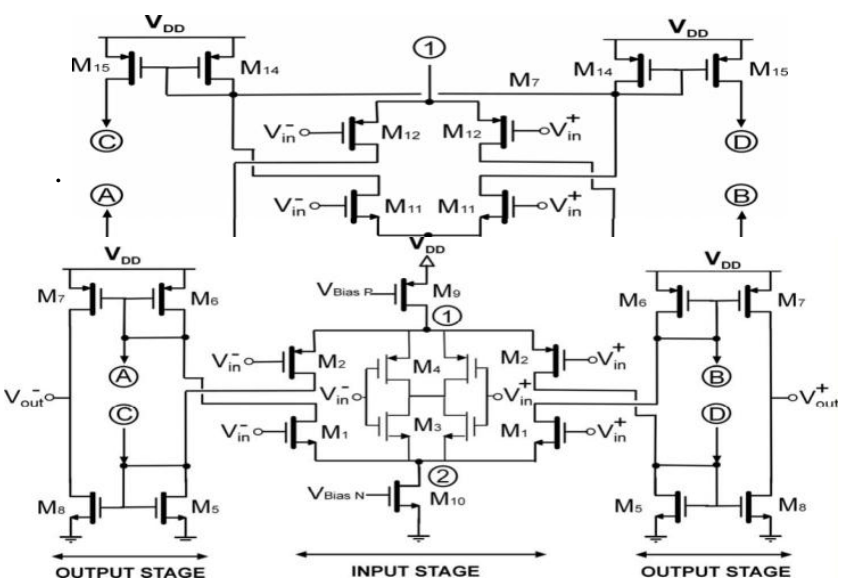

Fig.1 (b)

Figure 1 (a) Core of the proposed Operational Amplifier and (b) CMFF circuit.

\subsection{Rail to Rail op-amp based on Flipped Differential Pairs}

This CMOS operational amplifier [2] with constant transconductance is suitable for operation in low voltage environments. A constant transconductance is ensured for the whole common mode input stage which is based on flipped differential pairs. By utilization of an input stage composed by an NMOS and a PMOS flipped differential pair it is possible to get a high common mode excursion and to achieve at the same time constant transconductance gain and high CMRR. The proposed operational amplifier operates with a 1-V supply and is implemented in $0.18 \mu \mathrm{m}$ standard CMOS technology. The results show that a closed-loop unity-gain bandwidth achieves 7.6 $\mathrm{MHz}$ and an open-loop low-frequency gain is higher than $73 \mathrm{~dB}$ with a 1-M Ohm\| $15 \mathrm{pF}$ load.

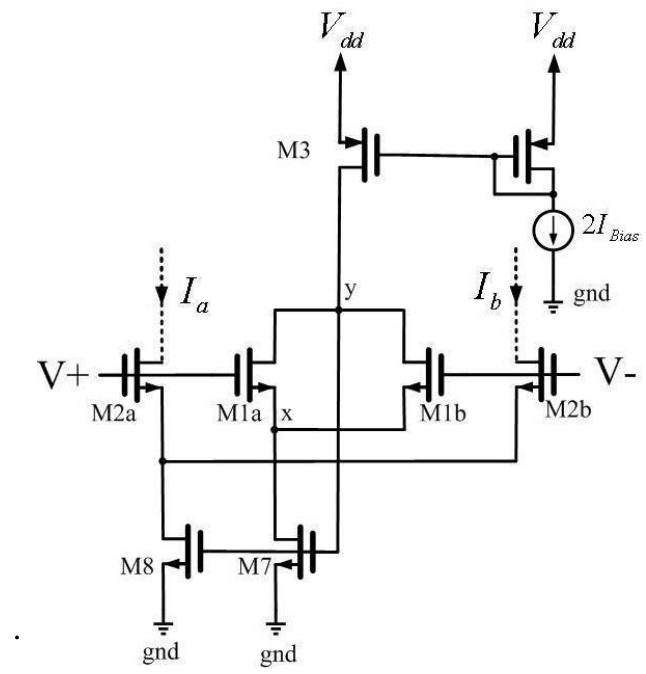

Figure 2 Flipped differential pair.

\subsection{Rail to Rail Operational Amplifier with Improved Transconductance Feedback Technique}

This op-amp [3] employs an improved transconductance feedback loop that senses the total transconductance (gm-T) accurately and enforces it to be equal to the conductance of a reference resistor. The traditional transconductance feedback technique equalizes the transconductance sum of the two transconductor stages to that of a reference stage in the feedback module. One drawback is that small gm-T fluctuation can be achieved only at the expense of high power consumption. This technique represents the input transconductance as small-signal resistance in diode-connected form and uses a reference resistor as the reference value. The proposed technique shows a significant improvement in power consumption while achieving a similar constant gm-T performance. Implemented in a $0.13-\mu \mathrm{m}$ standard CMOS technology under a $1-\mathrm{V}$ power supply, A folded cascode current summing stage followed by the class $A B$ output stage provides a open loop gain of $60 \mathrm{~dB}$ The op-amp achieves a unity-gain bandwidth of $3.7 \mathrm{MHz}$ with a 95-pF load while only consuming $187 \mu \mathrm{A}$ of quiescent current. 


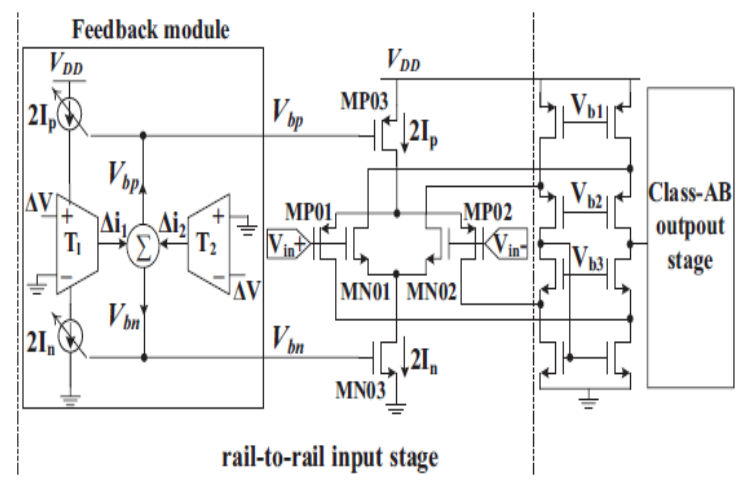

Figure 3 (a)

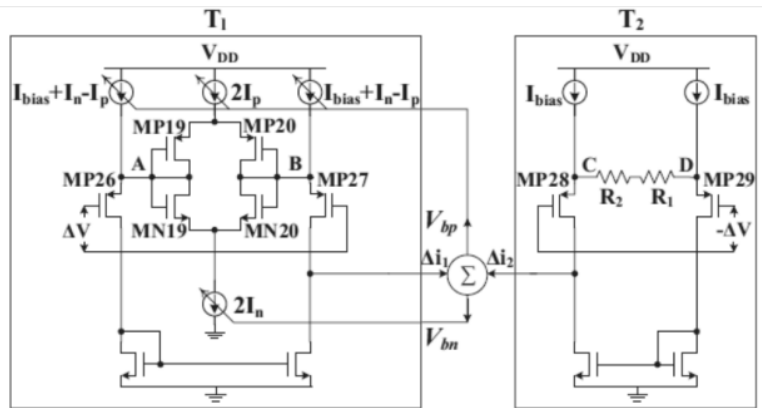

Figure 3 (b)

Figure 3 (a) Rail to Rail Amplifier structure (b) gm-T -R converter and resistive comparator in the feedback module.

\section{4. [PROPOSED WORK] 3x CURRENT MIRROR RAIL-TO-RAIL FOLDED CASCODE OPERATIONAL}

\section{AMPLIFIER}

This op-amp [proposed work] employs complementary input stage with a $3 \mathrm{x}$ current mirror technique for $\mathrm{Gm}$ regulation. Folded cascode architecture is proposed in part to improve the input common mode range, open loop gain and the power supply rejection of the two stage operational amplifier. It also has a classical common source output stage to achieve good input common mode range and the gain of the op-amp. The Common-Mode Feedback (CMFB) circuit insures that the drivers operate in true class A mode. The CMFB increases CMRR, reduces the variations in the output common mode voltage, controls the quiescent biasing conditions and keeps $\mathrm{V} 0 \mid \mathrm{CM}$ is kept almost constant. This work presents a design of a low-voltage CMOS rail-to-rail folded cascode operational amplifier realization in a standard $130 \mathrm{~nm}$ CMOS technology with $1.2 \mathrm{~V}$ supply voltage and consumes power less $<400 \mathrm{uW}$. A two stage folded cascode op-amp has a UGB $>10 \mathrm{MHz}$ and achieves a gain greater $>80 \mathrm{~dB}$ over almost full range of the common mode input voltage.

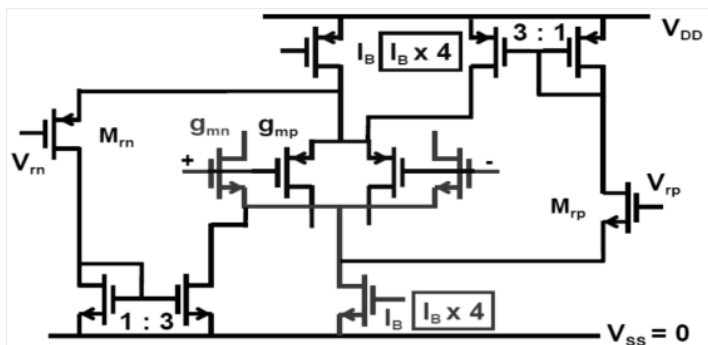

Figure 4 (a)

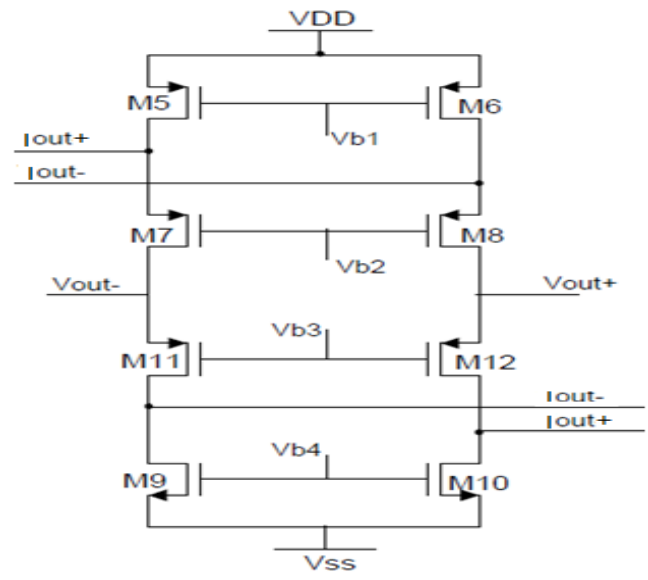

Figure 4 (b)

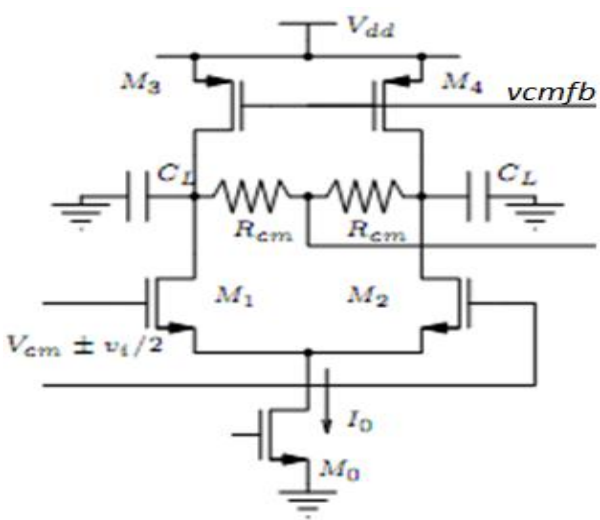

Figure $4(c)$

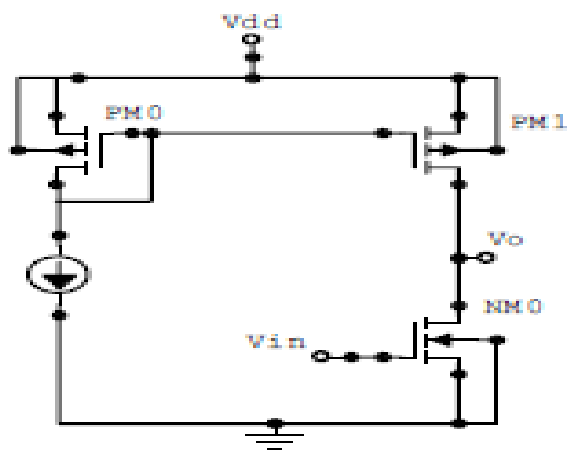

Figure 4 (d)

Figure 4 (a) $3 x$ current mirror Rail-to-Rail input stage structure (b) folded cascode summing stage (c) common mode feedback circuit (d) common source output stage 


\section{COMPARISON AND DISCUSSION}

Table 1

\begin{tabular}{|l|l|l|l|l|}
\hline Parameters & $\begin{array}{l}\text { Ref. } \\
\text { Paper } \\
\mathbf{1 .}\end{array}$ & $\begin{array}{l}\text { Ref. } \\
\text { Paper } \\
\text { 2. }\end{array}$ & $\begin{array}{l}\text { Ref. } \\
\text { Paper } \\
\text { 3. }\end{array}$ & $\begin{array}{l}\text { Proposed } \\
\text { work }\end{array}$ \\
\hline Technology & $\begin{array}{l}0.18 \\
\text { um }\end{array}$ & $\begin{array}{l}0.18 \\
\text { um }\end{array}$ & $\begin{array}{l}0.13 \\
\text { um }\end{array}$ & $\begin{array}{l}0.13 \\
\text { um }\end{array}$ \\
\hline $\begin{array}{l}\text { Supply } \\
\text { Voltage }\end{array}$ & $0.8 \mathrm{~V}$ & $1 \mathrm{~V}$ & $1 \mathrm{~V}$ & $1.2 \mathrm{~V}$ \\
\hline Gain & $51 \mathrm{~dB}$ & $73 \mathrm{~dB}$ & $60 \mathrm{~dB}$ & $>80 \mathrm{~dB}$ \\
\hline $\begin{array}{l}\text { UGB } \\
\mathrm{MHz}\end{array}$ & $\begin{array}{l}7.6 \mathrm{MHz} \\
\text { close- } \\
\text { loop) }\end{array}$ & $\begin{array}{l}3.7 \\
\mathrm{MHz}\end{array}$ & $\begin{array}{l}>10 \\
\mathrm{MHz}\end{array}$ \\
\hline $\begin{array}{l}\text { Phase } \\
\text { Margin }\end{array}$ & 64 & $65 \mathrm{Degree}$ & $\begin{array}{l}72 \\
\mathrm{Degree}\end{array}$ & $\begin{array}{l}>55 \\
\text { Degrees }\end{array}$ \\
\hline $\begin{array}{l}\text { Load } \\
\text { capacitance }\end{array}$ & $10 \mathrm{pF}$ & $15 \mathrm{pF}$ & $95 \mathrm{pF}$ & $10 \mathrm{pF}$ \\
\hline $\begin{array}{l}\text { Power } \\
\text { consumption }\end{array}$ & $4 \mathrm{uW}$ & - & $187 \mathrm{uW}$ & $<400 \mathrm{uW}$ \\
\hline
\end{tabular}

As we can see from the above comparison table the proposed 3x current mirror Rail-to-Rail folded cascode Operational Amplifier can provides high gain and unity gain bandwidth as compared to [1], [2], \& [3] op-amps. Proposed op-amp has relatively simple circuitry for $\mathrm{Gm}$ regulation as compared to the rest of the configuration.

\section{CONCLUSION}

Different methodologies for low voltage rail to rail op-amp design has been seen above. It shows that 'Rail to Rail op-amp Based on Flipped Differential Pairs' [2] and 'Rail-to-Rail Operational Amplifier with Improved Transconductance Feedback Technique' [3] has better Gm regulation as compare to [1] and proposed op-amp. Rail to rail op-amp with CommonMode Feed-Forward Circuit (CMFF) [1] less power consumption than rest of the techniques. The proposed ' $3 \mathrm{x}$ current mirror Rail-to-Rail folded cascode Operational Amplifier' can provides high open loop gain $>80 \mathrm{~dB}$ and unity gain bandwidth $>10 \mathrm{MHz}$ with the folded cascode scheme at the expense of higher power consumption.

\section{REFERENCES}

[1] M. R. Valero, S. Celma, N. Medrano, B. Calvo "A 4- $\mu$ W 0.8-V Rail-to-Rail Input/output CMOS Fully Differential OpAmp" Group of Electronic Design - I3A University of Zaragoza E-50009 Zaragoza, Spain. 978-14244-9137-7/11/\$26.00 @2011 IEEE

[2] Chao Guo, Siheng Zhu, Jun Hu, Jingjing Zou, Houjun Sun, Xin Lv,“ A Low Voltage CMOS Rail-to-Rail Operational Amplifier Based on Flipped Differential Pairs"School of Information Science and Technology, Beijing Institute of Technology, Beijing, China. 978-1-4244-8268-9/11/\$26.00 (C)2011 IEEE.

[3] Shanshan Dai, Xiaofei Cao, Ting Yi, Allyn E. Hubbard, and Zhiliang Hong, "1-V Low-Power Programmable Rail-to-Rail Operational Amplifier With Improved Transconductance Feedback Technique" IEEE transactions on very large scale integration (VLSI) systems, 1063-8210/\$31.00 @ 2012 IEEE

[4] Beal Rein Lerone, "A low voltage rail-to-rail operational amplifier with constant operation and improved process robustness" (2009).Graduate Theses and Dissertations. Paper 10718

[5] Hogervorst R. and Huijsing J. H.: Design of LowVoltage, Low Power Operational Amplifier Cells, Kluwer, 1996.

[6] Botma J. H., Wassenar R. F. and Wiegerink R. J.: Simple Rail-to-rail Low Voltage Constant Transconductance CMOS Input Stage in Weak Inversion, Electronics Letters, 1993.

[7] R.J. Baker, "CMOS - Circuit Design, Layout and Simulation", 2nd edition, IEEE Press, 2005.

[8] P.E. Allen and D. R. Holberg, "CMOS Analog Circuit Design”, 2nd edition, Oxford University Press, 2002

[9] B. Razavi, Design of analog CMOS integrated circuits, McGraw-Hill, 2000.

[10] D. Johns and K. Martin, "Analog integrated circuit design,” in John Wiley \& Sons, New York, 1997. 